\title{
BMJ Open Investigating the impact of headaches on the quality of life of patients with glioblastoma multiforme: a qualitative study
}

\author{
Samuel Robert Bennett, ${ }^{1}$ Garth Cruickshank, ${ }^{2}$ Antje Lindenmeyer, ${ }^{3}$ \\ Simon Rhys Morris ${ }^{1}$
}

To cite: Bennett SR, Cruickshank G,

Lindenmeyer $A$, et al. Investigating the impact of headaches on the quality of life of patients with glioblastoma multiforme: a qualitative study. BMJ Open 2016;6:e011616.

doi:10.1136/bmjopen-2016011616

- Prepublication history and additional material is available. To view please visit the journal (http://dx.doi.org/ 10.1136/bmjopen-2016011616).

Received 22 February 2016 Revised 25 October 2016 Accepted 27 October 2016

CrossMark

\begin{abstract}
${ }^{1}$ College of Medical and Dental Sciences, University of Birmingham, Edgbaston, Birmingham, UK

${ }^{2}$ Department of

Neurosurgery, Queen Elizabeth Hospital,

Edgbaston, Birmingham, UK

${ }^{3}$ Primary Care Clinical

Sciences, School of Health and Population Sciences, University of Birmingham, Edgbaston, Birmingham, UK
\end{abstract}

Correspondence to Samuel Robert Bennett; SRB157@bham.ac.uk

\begin{abstract}
Objectives: Headaches and facial pain have been identified as the most prevalent form of pain among patients with glioblastoma multiforme, the most common malignant primary brain tumour. Despite this, minimal research has been undertaken investigating the direct and indirect impact these headaches have on their quality of life. Therefore, in this study, we aimed at gaining a personal insight into the importance and impact that these headaches have on the quality of life of patients with glioblastoma multiforme.
\end{abstract}

Design: Exploratory study using face-to-face semistructured interviews. Interviews were audiorecorded, transcribed verbatim and then qualitatively analysed using thematic analysis.

Setting: Participants recruited from a tertiary referral hospital in Birmingham, UK.

Participants: Purposive sampling of 14 registered outpatients recently diagnosed with glioblastoma multiforme.

Results: 3 themes were identified: (1) an underlying attitude of determination and positivity; (2) impact of headache unpredictability on social interaction; (3) headaches found to act as a springboard onto thoughts regarding their disease and future.

Conclusions: While the quality of life of patients with glioblastoma multiforme is clearly multifactorial, headaches do indeed play a part for some. However, it is not the direct pain of the headache as one might expect that impacts on the quality of life of these patients, but the indirect effect of headaches through limiting patients' social lives and by serving as a painful psychological reminder of having a lifethreatening illness. In clinical practice, using headache diaries for these patients may help provide a more comprehensive assessment and further aid management plans. Alongside acting as an important reminder of the potential secondary implications of this disease, suggestions for future research include quantitatively investigating whether headaches can act as a prognostic indicator for quality of life within this patient demographic and determining whether these conclusions also hold true for a wider spectrum of patients with brain tumour.
Strengths and limitations of this study

- As far as the authors know this is the first descriptive exploratory study that has investigated the impact that headaches have on patients with aggressive brain tumours and the subsequent coping mechanisms that patients develop in response to this.

- The nature of the semistructured interviews and iterative process of analysis allowed emerging themes to be built on as the research progressed and hence develop a deeper understanding of the issues discussed.

- Family members present during the interviews may have had the potential to affect the way in which participants answered questions, especially when discussing such a personal and emotive subject as quality of life. However, overall it was seen as a benefit having them present as in most cases they aided in participant memory recall.

- Participation in the study was completely voluntary; hence, all participants were willing to talk about the impact of their symptoms on their quality of life-potentially missing patients who were eligible as far as the inclusion and exclusion criteria were concerned, but unwilling to discuss their quality of life and symptoms.

- All participants were newly diagnosed with a glioblastoma multiforme tumour and therefore the results may not be directly transferable to patients with recurrent glioblastoma multiforme who have had more time to process and live with their condition.

\section{BACKGROUND}

Glioblastoma multiforme (GBM) tumours are histologically the second most frequently reported primary brain and central nervous system tumour (accounting for 16\%) and the most common form of malignancy within this subset of neoplasms, ${ }^{1}$ with an annual incidence in the USA and Europe of 3 per 
100000 people. $^{2}$ The median age of diagnosis of this 2:1 male predominant disease is 64 years and it is the most aggressive form of the group of brain tumours known as astrocytomas (themselves a form of glioma). ${ }^{3}$ GBM tumours are categorised as a grade IV astrocytoma, stipulated by the WHO system, which grades astrocytomas from I to IV. ${ }^{4}$ Despite advances in treatment technology over the past few years, in particularly with regard to chemotherapy, ${ }^{5}$ due to the widely infiltrative nature and rapid growth of this tumour, associated life expectancy is still low, ${ }^{3}$ with a median survival of 15 months from diagnosis and a 2-year life expectancy ranging from $8 \%$ to $26 \%{ }^{6}$ To improve patient survival, standard treatment usually involves surgical debulking and biopsy of the tumour, followed by radiotherapy and chemotherapy. ${ }^{5}$ As a result of this poor prognosis in GBM, matters of quality of life (QoL) become of utmost importance to patients and those around them. ${ }^{7}$

The term QoL encompasses multiple aspects of a patient's overall well-being, including physical, material, social and emotional well-being. ${ }^{8}$ Over the past two decades, as survivorship of GBM has gradually increased and patients have survived longer to experience the comorbidities of associated treatments, ${ }^{6}{ }^{9}{ }^{10}$ QoL has become an ever more important factor in the management of such patients. Recommendations have been made for randomised trials to include QoL and palliative care outcomes as end points, ${ }^{11}$ although reliably measuring QoL in patients with high-grade glioma has proven difficult due to high rates of drop out bias and loss of participants' ability to complete complex forms. ${ }^{12}$

A significantly reduced overall QoL is seen in the majority of newly diagnosed patients with high-grade glioma, when compared with healthy controls. ${ }^{13} 14$ Descriptive research into the specific symptoms that affect QoL in patients with glioma has primarily focused on six manifestations: fatigue, sleep, pain, seizures, mood disturbance and cognitive function. ${ }^{15}$ However, despite headaches having been reported as the most prevalent form of glioma-associated pain, experienced by up to $52 \%$ of patients with $\mathrm{GBM},{ }^{16}$ most commonly as dull tension-type headaches, ${ }^{17}{ }^{18}$ and control of primary central nervous system tumour headaches being noted as crucial, ${ }^{19}$ no widely available or published research has investigated the impact that these headaches have on the QoL of patients with GBM. This may partly be due to the multifaceted complexity of investigating QoL and gaining patients' perspectives within this population.

Hence, with up to half of patients with GBM being affected by headaches,${ }^{16}$ if an association between headaches and QoL is identified, a deeper understanding of this could lead to the implementation of appropriate precautions or interventions, with the aim of improving the management of headaches in patients with GBM and subsequent QoL.

\section{AIIMS}

1. To establish an insight into the importance of headaches to patients with GBM.

2. To investigate the impact of headaches on the mindset of patients being treated for an aggressive brain cancer.

\section{METHODS}

Design

This exploratory qualitative study employed face-to-face semistructured interviews with patients being treated for GBM at the Queen Elizabeth Hospital Birmingham (QEHB) -all of which were interviewed between 5 and 21 weeks postradiotherapy. At the time of the interview, participants were at various stages of their adjuvant temozolomide chemotherapy cycle, with each participant only interviewed once. The purpose of selecting this time period was to interview participants in a position where they felt they had enough experience to talk and reflect about their condition, symptoms and associated QoL. Additionally, all participants were required to be over the age of 18 at the time of being informed of the study. Potential participants with a WHO Performance Status $>2$ (not capable of self-care) were excluded from the study due to the severity of their illness. Owing to this study being the first of its kind in this specific area of research, use of questionnaires and a quantitative approach was dismissed as it was felt this may miss out on in-depth specific details regarding QoL. When investigating the complexity of human behaviour, a qualitative approach may more likely highlight these due to the themes that emerge when participants are allowed to discuss what is important to them. ${ }^{20}$ Such an approach has been successful in similar research in patients with other cancers and congenital heart disease. ${ }^{21} 22$ Additionally, due to the limited time frame and resources, the feasibility of recruiting a sample size capable of statistical significance from questionnaire data would have been low.

\section{Sample size}

Of the 21 eligible participants, identified from neuro-oncology clinic lists and informed of the study using purposive sampling, 14 were subsequently interviewed within the Cancer Centre Department of the QEHB. Of the seven who were not interviewed, four were because of late hospital appointment changes, with only three actively declining to take part.

\section{Data collection}

Over a 5-month period (February to April, July and September 2015) potential participants were identified and informed of the study by a MacMillan Clinical Nurse Specialist in their existing care team. This was carried out either face-to-face or by telephone, using a participant information sheet to explain the purpose of the research, with interviews scheduled to coincide with 
their next outpatient appointment at the QEHB. The interviews, each lasting 15-40 min, were undertaken by a senior medical student (SRB), as part of a qualitative focused intercalated research degree, following a prewritten interview topic guide (see online supplementary file 1). This topic guide was designed by the research team (SRB and AL) to explore both headache and nonheadache participants' views regarding the changes in their life and symptoms experienced in the build up to and since their diagnosis of GBM. This specifically focused on QoL and the impact that headaches or the potential for headaches and what they had now come to represent had on this. The reasoning for asking additional questions, not just related to headaches, was so that the interviewer could immerse themselves and grasp as much as possible regarding the personal challenges that these patients now face-in turn allowing for a more comprehensive understanding and analysis of the impact and role that headaches play within this. Owing to the small-scale nature of the study and time restrictions, the topic guide was not pilot tested with participants; however, the semistructured nature of these interviews and iterative process of analysis allowed a continuous adaption of the interview guide throughout the study, so as to build on emerging themes. As the study progressed, no questions were added or removed from the interview guide, but greater emphasis was placed on the thoughts and feelings that participants associated with headaches. No field notes were made during the interviews; however, each interview was transcribed as soon as possible so as to retain non-verbal information that the interviewer identified during the interview.

\section{Data analysis}

Data were analysed following Braun and Clarke's 6 Step Thematic Analysis, allowing identification and analysis of patterns and themes. ${ }^{23}$ The interviews, audio-recorded digitally, were transcribed verbatim (including notes on participant body language), with transcripts read and re-read to ensure familiarity, before deriving codes. Once interviews were coded, they were then collated to generate themes and extract important issues, viewpoints and dynamics, which led to a subsequent active data search for disconfirming evidence. ${ }^{21}$ Analysis of derived codes and theme recognition was carried out independently by the interviewer (SRB), a lecturer in qualitative methods (AL) and an additional senior medical student (SRM), to identify and understand potential multiple interpretations of the data and reduce the likelihood of producing results which were partial or biased. ${ }^{24}$ This theme identification began before data collection was complete in order to explore initial findings in greater depth. All research activities were recorded in detail to allow methodological critical appraisal and increase trustworthiness, a concept used to describe reliability in qualitative research. ${ }^{25}$

However, due to the limited time frame, further validity checking using participant verification was not feasible.
Interviews were selected over focus groups due to the highly sensitive nature of discussing QoL within this population, where participants may have felt reluctant to divulge such personal information in front of strangers. However, a family member was allowed to contribute to the interview, if requested so by the participant.

\section{Research ethics}

All patients who took part in this study participated voluntarily. Written informed consent was taken from all participants prior to starting each scheduled interview, which were all undertaken within a quiet confidential setting in the Cancer Centre, QEHB. Participants were also informed that they could stop the interview at any point for any reason. All confidential participant information was stored on encrypted memory sticks, accessible only to the researchers.

\section{RESULTS}

Of the 14 participants (median age $=47.5$ (IQR 38-63) years; 8 male) interviewed, 6 reported as experiencing headaches within the last month, 1 reported a unilateral facial pain and 1 other with lightheadedness, but no headaches (table 1). The remaining six patients reported as having neither headaches nor facial pain nor lightheadedness. All participants reported varying levels of tiredness and fatigue. Additionally, all participants requested to have a family member or carer present during the interviews.

Grouping and interlinking of codes, derived from all of the transcribed interviews, yielded three themes, however only the first two of these, 'underlying determination and positivity' and 'headache of social situations', were initially apparent. These themes primarily describe the thoughts, feelings and emotions conveyed by the participants, regarding their approach to the challenges that day-to-day life now presents and the role of headaches within this. Only after full data immersion and code collaboration was the third theme, 'psychological springboard of headaches', developed. This final theme aims to provide an insight into the potential implications that headaches, and what they have now come to represent, may have on self-contemplation in this population regarding their condition and future. Of these themes, 'underlying determination and positivity' was developed from all participants (both headache and non-headache), with the remaining two themes developed only from the six participants who developed headaches and the two participants who reported facial pain and lightheadedness.

\section{Underlying determination and positivity}

A consistent theme among almost all participants was a resounding determination to 'get on with life' and not to be bound by the symptomatic implications of their condition, referred to by several as 'the nature of the beast': 
Table 1 Participant information

\begin{tabular}{llll}
\hline $\begin{array}{l}\text { Participant } \\
\text { number }\end{array}$ & Gender & $\begin{array}{l}\text { Number of weeks interviewed } \\
\text { postradiotherapy }\end{array}$ & $\begin{array}{l}\text { Headaches experienced within the last } \\
\text { month }\end{array}$ \\
\hline 1 & Male & 12 & Yes \\
2 & Female & 8 & Yes \\
3 & Male & 8 & Yes \\
4 & Female & 5 & Yes \\
5 & Female & 13 & No \\
6 & Female & 13 & Yes \\
7 & Female & 12 & No \\
8 & Male & 5 & No (but facial sensitivity/pain) \\
9 & Male & 5 & Yes \\
10 & Male & 20 & No \\
11 & Male & 15 & No (but lightheadedness) \\
12 & Male & 8 & No \\
13 & Male & 18 & No \\
14 & Female & 17 & No \\
\hline
\end{tabular}

I try not to think about it, which may not be the healthiest psychological way to go about it. However, it just means I can get on throughout the day, getting on with things that I like to do and want to do. (P2)

Acceptance of the situation, alongside the want and almost need to block it out in order to carry on with as normal a life as possible, was a commonly reported trait. Potential reasoning to partially explain this behaviour can be drawn from the frequently reported frustration that came with participant's self-recognition of dwelling on their illness:

obviously I think about the future, because I know it's not curable, it's a controllable cancer...it's a bit frustrating if you think about it too much, but then you've got to be thankful for what you've still got. (P5)

While this theme is not directly related to headaches, the principle of leading as normal life as possible was the fundamental aspect of most of the participants' approach to their brain tumour, expressed by 12 of the 14 interviewed. With this in mind, this then helps contextualise the next two themes, discussing ways in which headaches were found to disrupt normality.

\section{Headache of social situations}

While not all participants reported as having headaches, three of the six who did noted the subsequent impact on their social lives:

I do kind of think if I'm on good form I'll be alright and it'll be fun, but if I don't feel good it won't be fun...I don't really know what to say or how to join in. (P4)

For this participant, the key issue with group interaction was that her symptoms of headaches and fatigue were experienced unpredictably and when they were experienced 'you kind of feel like you're a bit disconnected, like you can't think properly and it's hurting'.
While admitting that the headaches she experienced were not 'debilitating', they or the concern of them had prevented her from so far socialising with groups of friends. Similar reservations regarding socialising in a group were also reported by other participants:

I'm very happy to see my friends one-to-one, but the thought of seeing people in a group, I don't know if I feel that's a bit more intimidating than I would have done beforehand. (P2)

While feeling comfortable socialising one-to-one with people, this participant expressed worry about interacting in a group in case they started having headachestheir concern stemming from the fact that they would not then want the rest of the group to start worrying about them.

\section{Psychological springboard of headaches}

The final theme that emerged was an awareness, and in some cases fear, of the negative connotations that headaches had now become associated with. When feeling 'tired and headachey', several participants reported it made them think 'oh, what is going on in my head' and acted as a springboard onto subsequent negative thoughts regarding their condition and future:

You start to think "oh is there something else going on upstairs". (P11)

Here, this participant talked about the consequent effect on thought process and rationale that their lightheadedness and frequent twinges in their forehead now held for them. While they expressed their determination to maintain a positive outlook, these symptoms were often an untimely and certainly unwanted reminder of their cancer.

As identified in the first theme, a positive mentality was the overwhelming attitude conveyed by the 
participants. To maximise this positive outlook and avoid these psychological reminders of their condition, participants voiced that they had adapted their lives to minimise the chance of headaches:

The only thing that I don't do is drink alcohol anymore, and that's not because I'm not allowed to...It's probably the fact that I don't want to have a glass of wine if that's going to give me a headache. (P6)

With this participant, if headaches were to become more regular, she admitted she would assume the worst and think 'oh, it's growing and growing and growing and taking over my head', even if the headache aetiology could clearly be associated with some far more benign behavioural activity (such as caffeine or alcohol). Similar participant reflection revealed an insight into how headaches may not only act as a psychological springboard but also as a further symptomatic springboard:

They may possibly have been part of the reason that I wasn't sleeping properly, because, they were part of the reason I wasn't feeling great. (P3)

While this participant was confident that the sporadic headaches he had been experiencing were not the cause of his waking at night, they may have contributed to him struggling to fall back asleep, leading to not only contemplation of his condition and future at night, but also to day time tiredness, subsequently limiting activities of daily living. This is a crucial point, as the double-edge of cancer is that it not only limits life expectancy, but also limits the amount that patients are capable of doing in this remaining time. In terminal cancer, this is even more so relevant, where treatment often shifts towards prioritising QoL.

\section{DISCUSSION}

During analysis, three themes emerged: 'underlying determination and positivity', 'headache of social situations' and 'psychological springboard of headaches'. While most participants agreed that headaches themselves did not cause great direct physical pain, and would only start to worry about headache pain more if they were 'more prolonged' or 'severe', this may have been affected by the symptoms experienced in the run up to diagnosis, of which several had severe headaches. Owing to previous experience of severe headaches, diagnosis of GBM and adequate time to reflect on their situation, the emotional and psychological impact of headaches and coming to terms with their condition was more often ( $\mathrm{n}=5$ of 6 headache participants) found to be greater than their physical sequela.

Furthermore, a seemingly key aspect within this population, to maintaining QoL and partaking in normal activities of daily living, was to focus on the positive and not the negative aspects of their lives-with participants reporting both actively trying not to think negatively, and passively by immersing themselves in hobbies, socialising, travelling or even returning to work. This participant behaviour of seeking normality and attempts to distance themselves from the taboo of cancer has been previously identified in similar qualitative interviews focusing on the QoL of patients with anal cancer. ${ }^{21}$ However, when symptoms were experienced and impacted on day-to-day life, it became harder for participants to forget about their condition. Within this, some symptoms were seen to be associated with fewer negative connotations, for example, tiredness and fatigue, to which participants reported incorporating increased rest and daytime sleep into their daily routine, but did not tend to think too much into them. However, headaches had more scope to play on the mind (reported by four of the six participants with headaches). This may be due to the more direct and potentially easier link to psychologically make between headaches and the knowledge of having a brain tumour, than fatigue and a brain tumour. Hence, the presence of headaches may make the ability to forget about the situation that these patients are facing harder, subsequently indirectly impacting on QoL. Additionally, of the eight participants who reported not having headaches, five voiced that if they did start experiencing headaches, they would likely jump to conclusions and panic about the aetiology.

\section{Recommendations}

Potential interventions could include transferring lifestyle management strategies from the standard treatment of tension-type headaches, such as keeping a headache diary to identify triggers or exacerbating factors as well as aiming to relieve patients with GBM of the worry surrounding the aetiology of their headaches ${ }^{26}$ however, a downside of this could be even further contemplation of their condition. While it was felt that saturation was reached in this study (with themes becoming repetitive by the latter stages and no new themes emerging), if similar qualitative research was to be conducted, assessing whether the results of this study also holds true for a range of other malignant or even benign brain tumours, a larger sample size would be recommended if multiple disease pathologies were to be allowed within the inclusion criteria. It could also be investigated whether headaches can be used to predict QoL within this population, by undertaking a prospective longitudinal study aiming to identify whether the presence of headaches immediately postradiotherapy (when QoL is considered to be at its worst) can act as a prognostic indicator for QoL during the adjuvant phase of treatment. Validated questionnaires, such as the European Organisation for Research and Treatment of Cancer Quality of Life Questionnaire C30 and BN20, could be provided to patients postradiotherapy and then again several months later. Here, an upward trend of QoL would be expected, with this proposed study aiming to identify whether the presence of headaches affects the trajectory of this upward curve. 


\section{Limitations}

Limitations include a small sample size (however, this is normal for qualitative research) and family present during the interview having the potential to affect participants' answers. In addition, participation in the study was completely voluntary; hence, all participants were willing to talk about the impact of their symptoms on their QoL ( potentially missing patients who were eligible as far as the inclusion criteria, but unwilling to discuss their QoL and symptoms). Additionally, participants interviewed within this study were all undergoing treatment for a newly diagnosed GBM tumour and hence the results may not be transferable to patients with recurrent GBM. Participants were either undergoing or had recently undertaken adjuvant temozolomide in the chemotherapy phase of their treatment; however, the exact phase of the chemotherapeutic cycle that they were in at the time of the interview was not recorded and neither was their current medication regime. Initially, inclusion criteria stipulated that patients be 5-9 weeks postradiotherapy at the time of interview; however, due to the limited time period of the study, it was realised this would not be feasible to recruit enough participants and hence was expanded to 5-21 weeks. A further difficulty encountered was classifying exactly who had headaches, with one participant reporting a unilateral facial pain and another with pronounced lightheadedness; however, both were quite adamant they were not experiencing headaches and as such were recorded that way. Memory recall of headaches experienced was not seen as a limitation, as when a participant was unsure of the timings of their headaches, they were offered help by their family member in attendance.

Owing to the multifaceted complexity that QoL presents in patients with brain tumour, research specifically focusing on the burden of just one symptom that plays a role in this is challenging. While headaches are not the most prevalent symptom experienced by this population, they are indeed experienced by a significant proportion. Through identifying themes consistent among patients with GBM with headaches, we seek to gain a deeper understanding of the day-to-day challenges that this cohort faces, with the aim of further facilitating patient management.

Despite these limitations, the principles raised in this study may possibly be applicable, at least to varying extents, to a larger population of not just patients with GBM, but potentially also other patients with high-grade malignant and terminal brain cancer or even patients with benign brain tumours experiencing headaches.

\section{CONCLUSION}

The aim of this qualitative study was to investigate and address the impact, importance and interaction of headaches experienced by patients with GBM, on their QoL. While not all participants reported as experiencing headaches, those that did considered the physical pain of their headaches not to be severe or prolonged enough to directly impact on QoL. However, during interview analysis, three participant themes emerged regarding the strive to not be bound by the symptomatic implications of their condition, the indirect impact of headaches on socialisation and headaches acting as a painful psychological reminder in certain participants of the significance of the threat to their autonomy and survival. These emergent themes should act as important reminders to clinicians of the secondary impacts and underlying mindset of patients having been diagnosed or undergoing treatment for GBM, in particularly if they have been experiencing headaches. Implications for clinical practice include the potential for providing patients with headache diaries to allow a more comprehensive holistic assessment of patients with GBM experiencing headaches. Directions for further research include investigating whether headaches can be of clinical value by being used as a prognostic indicator for QoL and exploring if the themes raised in this study also bear relevance to subsets of patients with a wider spectrum of brain tumours.

Acknowledgements The authors thank Dr Jon Ives, Dr Deirdre Lane and Edward Hutchison for their advice and assistance during the planning phases of this study, MacMillan Neuro-oncology Clinical Nurse Specialists Claire Goddard and Fred Berki for their invaluable continued support during participant recruitment and throughout the study, Valerie Dodd for providing an inspiration to those around her and most importantly, to the participants, who without their time, this study would not have been possible. This research was presented as a poster at the 2015 Society for Neuro-Oncology conference (San Antonio, Texas, USA).

Contributors This study was devised by SRB and subsequently designed together with GC and AL. SRB interviewed, transcribed and provided subsequent coding. Thematic development of these codes was performed independently by SRB, AL and SRM, to which SRB then collaborated with both, agreeing on the final three key themes. SRM wrote the first drafts of the paper, which were then critiqued and edited by GC, AL and SRM-with the final version approved by all.

Funding This research received no specific grant from any funding agency in the public, commercial or not-for-profit sectors.

Competing interests None declared.

\section{Patient consent Obtained.}

Ethics approval This research was approved by the 'West Midlands-Solihull' National Research Ethics Committee (15/WM/0012) with subsequent site-specific approval from the QEHB Research and Development Department.

Provenance and peer review Not commissioned; externally peer reviewed.

Data sharing statement No additional data are available.

Open Access This is an Open Access article distributed in accordance with the Creative Commons Attribution Non Commercial (CC BY-NC 4.0) license, which permits others to distribute, remix, adapt, build upon this work noncommercially, and license their derivative works on different terms, provided the original work is properly cited and the use is non-commercial. See: http:// creativecommons.org/licenses/by-nc/4.0/

\section{REFERENCES}

1. CBTRUS. CBTRUS Statistical Report: primary brain and central nervous system tumors diagnosed in the United States in 20042008. 2012:15. (23 March 2012 Revision. http://www.cbtrus.org/ 2012-NPCR-SEER/CBTRUS_Report_2004-2008_3-23-2012.pdf (accessed 20 Oct 2014).

2. Adamson C, Kanu OO, Mehta Al, et al. Glioblastoma multiforme: a review of where we have been and where we are going. Expert Opin Investig Drugs 2009;18:1061-83. 
3. Lindsay K, Bone I, Fuller G. Neurology and neurosurgery illustrated. 5th edn. Churchill Livingstone, 2010:316-17.

4. Louis DN, Ongaki H, Wiestler OD, et al. The $2007 \mathrm{WHO}$ Classification of tumours of the central nervous system. Acta Neuropathol 2007;114:97-109.

5. Zhang X, Zhang W, Cao WD, et al. Glioblastoma multiforme: molecular characterization and current treatment strategy (review). Exp Ther Med 2012;3:9-14.

6. Nagasawa DT, Chow F, Yew A, et al. Temozolomide and other potential agents for the treatment of glioblastoma multiforme. Neurosurg Clin N Am 2012;23:307-22.

7. Cheng JX, Zhang X, Liu BL. Health-related quality of life in patients with high-grade glioma. Neuro Oncol 2009;11:41-50.

8. Felce D, Perry J. Quality of life: its definition and measurement. Res Dev Disabil 1995;16:51-74.

9. Rajan B, Ross G, Lim CC, et al. Survival in patients with recurrent glioma as a measure of treatment efficacy: prognostic factors following nitrosourea chemotherapy. Eur J Cancer 1994;30a:1809-15.

10. Marijnen CA, van den Berg SM, van Duinen SG, et al. Radiotherapy is effective in patients with glioblastoma multiforme with a limited prognosis and in patients above 70 years of age: a retrospective single institution analysis. Radiother Oncol 2005;75:210-16.

11. Gupta T, Sarin R. Poor-prognosis high-grade gliomas: evolving an evidence-based standard of care. Lancet Oncol 2002;3:557-64.

12. Henriksson R, Asklund T, Poulsen HS. Impact of therapy on quality of life, neurocognitive function and their correlates in glioblastoma multiforme: a review. J Neurooncol 2011;104:639-46.

13. Taphoorn MJ, Sizoo EM, Bottomley A. Review on quality of life issues in patients with primary brain tumors. Oncologist 2010;15:618-26.
14. Brown PD, Ballman KV, Rummans TA, et al. Prospective study of quality of life in adults with newly diagnosed high-grade gliomas. $J$ Neurooncol 2006;76:283-91.

15. Liu R, Page M, Solheim K, et al. Quality of life in adults with brain tumors: current knowledge and future directions. Neuro Oncol 2009;11:330-9.

16. Osoba D, Brada M, Prados MD, et al. Effect of disease burden on health-related quality of life in patients with malignant gliomas. Neuro Oncol 2000;2:221-8.

17. Schankin CJ, Ferrari U, Reinisch VM, et al. Characteristics of brain tumour-associated headache. Cephalalgia 2007;27:904-11.

18. Forsyth PA, Posner JB. Headaches in patients with brain tumors: a study of 111 patients. Neurology 1993;43:1678-83.

19. Purdy RA, Kirby S. Headaches and brain tumours. Neurol Clin 2004;22:39-53.

20. Agius SJ. Qualitative research: its value and applicability Psychiatrist 2013;37:204-6.

21. Mortensen GL, Lundby L. Patients use normalisation techniques to cope with the quality-of-life effects of anal cancer. Dan Med $J$ 2015;62:A5020.

22. Claessons $\mathrm{P}$, Moons $\mathrm{P}$, de Casterle $\mathrm{BD}$, et al. What does it mean to live with a congenital heart disease? A qualitative study on the lived experiences of adult patients. Eur J Cardiovasc Nurs 2005;4:3-10.

23. Braun V, Clarke V. Using thematic analysis in psychology. Qual Res Psychol 2006;3:77-101.

24. Booth A, Carroll C, llott I, et al. Desperately seeking dissonance: identifying the disconfirming case in qualitative evidence synthesis. Qual Health Res 2013;23:126-41.

25. Guba EG. Criteria for assessing the trustworthiness of naturalistic inquiries. Educ Resour Inf Center Ann Rev Paper 1981;29:75-91.

26. Heachache: MedlinePlus Medical Encyclopedia. http://www.nlm.nih. gov/medlineplus/ency/article/003024.htm (accessed 13 Nov 2014). 